\title{
THE RESPONSE BEHAVIOUR OF ANT PheIDologeton diversus ON ENCOUNTERING AN OBSTACLE ALONG ITS TRAIL
}

\author{
Vijay Mala Nair
}

Senior lecturer, Department of Applied Zoology, Mangalore University, Mangalagangotri, Karnataka 574199, India.

\begin{abstract}
The response behaviour of ant, Pheidologeton diversus was studied following interruption of the trail line with an elongate and a circular object across its path. It was observed that ants could not continue to move along the trail line in case of both the obstructions causing chaos and crowding at the point of intersection with the object. However, in the case of the linear object they were found to rejoin the broken trail by creating two distinct paths, one parabolic and another straighter one with a acute $\mathrm{U}$ turn along the object. In both cases, the paths were created towards the shorter section of the obstacle. While in the case of the circular object, a semicircular path was formed along the circumference of the object. There was no distinct bifurcation as in the case of the linear object.
\end{abstract}

\section{Keywords}

Ant, Pheidologeton diversus, encountering obstacle, trail line, selection of path

\section{Introduction}

The main means used by ants to form and maintain the line is a pheromone trail. The trail laying and trail following seen in mass recruitment constitute a simple allelomimetic decision process and autocatalytic process (Holleidobler \& Wilson, 1990; Goss et al., 1989). A study carrying series of laboratory experiments have shown that colonies of the ant, Lasius niger select the shorter of the two routes (of varying length, form and in different planes) available between nest and foraging area (Beckers $e t$ al., 1992). Ants are known to find a new shortest path on encountering a new obstacle, which interrupts the initial path (Beckers et al., 1992 \& Goss et al., 1989). The present study records the response behavior of the ant, Pheidologeton diversus moving to and fro carrying food to their nest after encountering the sudden appearance of an unexpected obstacle of different shapes across their already established natural trail line.

\section{Materials and Methods}

A natural ant line of Pheidologeton diversus found in front of a house moving to and fro from nest to food source was obstructed by placing objects of different shapes. In the first case a linear object (length $19 \mathrm{~cm}$ and diameter $7.5 \mathrm{~mm}$ ) was placed in the path of the ant line, such that the projecting part of the object dividing the ant line was of a shorter $(3.0 \mathrm{~cm})$ and longer length $(16 \mathrm{~cm})$ length on either side of the line (Fig. 1). Observations were also recorded after placing exactly similar object of same dimensions approximately three feet from the previous object in the reverse order simultaneously as shown in Fig. 2. In the second case observations were recorded after placing a circular object (circumference of $13.6 \mathrm{~cm}$ ) across the path of ants aligning the trail line with the midline of the object (Fig. 2).

\section{Results and Discussion}

It was observed that, Pheidologeton diversus could not continue to move along the trail line for a while causing chaos and crowding at the point of obstruction.

The ants carrying food towards the nest initially seemed to lose direction for a while, but soon they were observed to rejoin the trail line at the shorter side of the obstacle turning around to the other side of the obstacle taking a parabolic course (Fig. 1 \& 2). In contrast, the ants moving towards the food source, carrying no food were noticed moving at a slower pace along the length of the obstacle trying to find a way and were soon found to rejoin the broken trail line by making a more acute U-turn (Fig. 1 \& 2) at the end of the shorter section of the obstacle. Reconstitution of the interrupted line selecting the shorter path around the obstacle seems to be an autocatalytic process (Holldobler \& Wilson, 1990). Most of them (about 98\%) did not cross over the object to rejoin the trail, perhaps the shortest path possible in principle 


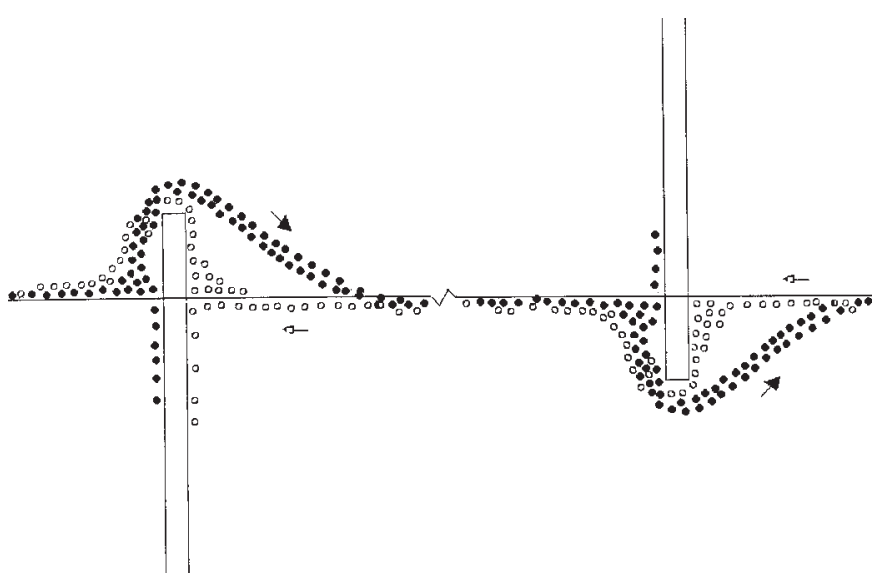

Figure 1. The diagrammatic representation of the response behaviour of ant, Pheidologeton diversus on placing a linear obstacle perpendicular to its trail line. Note the ants carrying food moving nest ward (arrow) represented by a solid circle (s) followed a parabolic path in contrast to the ones not carrying food represented by an open circle (o) moving towards the food source

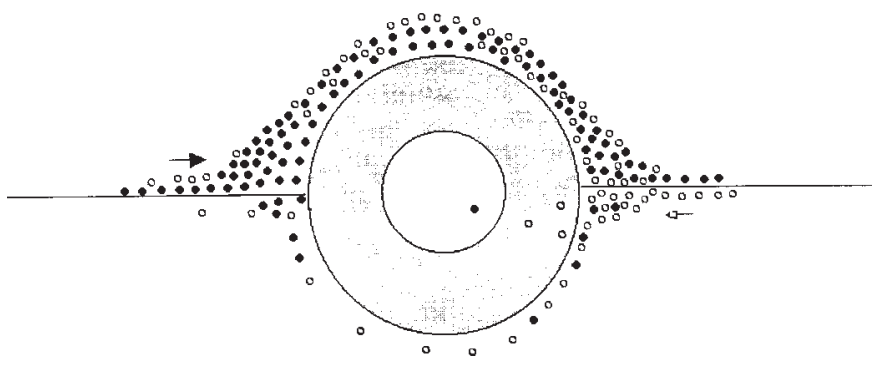

Figure 2. The diagrammatic representation of the response behavior of ant, Pheidologeton diversus on placing a circular obstacle across its trail line. Note both the ants carrying food moving nest ward (arrow) and the others moving towards the food source represented by a solid circle and an open circle (o) respectively followed a semicircular path.

The encountering of an obstacle by the ants in the present study led to the bifurcation of the original trail into two distinct path lines for a short distance around the obstacle one consisting of a parabolic path taken predominantly by ants carrying food and the other by the ants moving towards the food source respectively (Fig. 1). However, in the case of the circular object, the ants were observed to rejoin the broken trail line on reaching halfway along the circumference of the circular object moving along the boundary making a semicircular path. (Fig. 2). The parabolic /acute $U$ turn path in the case of a linear object and a semicircular path in the case of a circular object seems to be an emergent property of the interaction between the obstacle shapes.

It is interesting to note that in the case of a linear object, the parabolic path followed for a short distance by the ants carrying food is indeed shorter than the U turn path, around the object, followed by ants not carrying any food at all considering the total length traversed by ants between the nest and food source. The observed difference in behaviour noted in the present study may perhaps relate to the objective of the movement.

Selecting the shortest path is of great adaptive value to an animal that uses chemical recruitment trails to exploit large resources in terms of the quantity of food retrieved (Traniello, 1987). The ants not carrying food did not follow the new parabolic path constructed by ants carrying food. They however, continued to course along the older trail line till they reach the obstacle and moved further taking an acute U-turn by going around the obstacle. Moffett (1987) has suggested that the ant, Pheidologeton diversus is able to determine the direction by their nest mates, which are laden with food. It is intriguing in the present study, that the ants returning from nest after dropping the food choose the straighter path despite having the choice of following the parabolic path laid by ants carrying food to the nest (Fig. 1). However, there is a need to further investigate the choice of trail following by ants.

The present study also points that the initial selection of shorter path at the end of the obstacle differs among ants carrying food and the ones not carrying food, which aggregated at the shorter end of the obstacle, leading to the formation of a parabolic and acute U-paths respectively. Is this selection process induced by the weight of the object being carried requiring conservation of energy? This question requires further investigation.

\section{References}

Beckers, R., J.L. Deneubourg and S. Goss (1992). Trails and Uturn in the selection of the shortest path by the ant, Lasius niger. Journal of Theoretical Biology 159: 397-415.

Goss, S., S. Arnon., J.L. Deneubourg and J.M. Pasteels (1989). Self organized shortcuts in the Argentine ant. Naturwissenchaften 76: 579-581.

Holldobler, B. and E.O. Wilson (1990). The Ants. Springer-Verlag, Berlin

Moffett, M.W. (1987). http://www.psychology.nottingham.ac.uk/resea rch/aarg/ant/project.html

Traniello, J.F.A. (1987). Comparative foraging ecology of North Temperate ants: the role of worker size and cooperative foraging in prey selection. Insectes Sociaux 34: 118-130. 